\title{
Lung function, genetics and ethnicity
}

\author{
Peter Burney and Richard Hooper ${ }^{2}$
}

\begin{abstract}
Affiliations: ${ }^{1}$ Wellcome Centre for Global Health, MRC-PHE Centre for Environment and Health, National Heart and Lung Institute, Imperial College London, London, and ${ }^{2}$ Centre for Primary Care and Public Health, Blizard Institute, Queen Mary University of London, London, UK.
\end{abstract}

Correspondence: P. Burney, Respiratory Epidemiology, Occupational Medicine and Public Health, Emmanuel Kaye Building, Imperial College London, 1 Manresa Road, London, SW3 6LR, UK.

E-mail: p.burneyđimperial.ac.uk

Professor Quanjer's editorial makes several errors in reporting our views on ethnicity and lung function [1], views that had been fairly summarised by BRAUN et al. [2] in the paper on which the editorial comments. Among his more unfortunate assertions is that our conclusions are reminiscent of the racist views of Hoffman. As the purpose of the paper to which the editorial refers was precisely to question the residual support that current assumptions provide for a racial explanation of ethnic differences [3], this seems particularly inappropriate.

The observed differences in vital capacity between different ethnic groups raise two principal questions, "What accounts for these differences?" and "What implications do they have for health?" The first question is unanswerable on the current evidence but the prevalent view, and one that would seem to be encouraged by Prof. Quanjer's peroration on the development of genetic diversity, is that it is explained by genetics. As Prof. Quanjer himself points out, however, associations are not necessarily causal, and low ventilatory function is also strongly associated with poverty.

Disentangling the contributions of genetics and poverty has not yet been possible. One technique used recently is liable to be over interpreted. Recognising that gene pools are increasingly mixed and that genetic structure may confound the interpretation of studies, geneticists have developed methods for quantifying genetic ancestry from the genome [4]. These methods are essential for adjusting for likely confounding in genetic studies, but they cannot be used to demonstrate a genetic origin for associated phenotypes. The greater the "African" component of the genome, the lower the lung function [5,6], but this does not imply that the cause of the low lung function in people with an African heritage is genetic. These observations show only that ethnicity and ventilatory function are related; they further quantify the initial question but do not answer it. Among people of African origin, social conditions such as job, income and education are associated with darkness of skin (presumably largely determined by genes) [7], but we do not assume that these social disadvantages are explained simply by genetic inheritance. Even where differences in lung function exist after adjustment for some social factors [6] the possibility of residual confounding exists. To establish a genetic explanation for the differences will require identification of specific polymorphisms that are responsible for the ethnic differences.

There is equally good evidence that at least chronic obstructive pulmonary disease (COPD) mortality is strongly associated with poverty even among fairly homogeneous populations [8]. In the UK the social class gradient for deaths from COPD in 1980 was far steeper than the social class gradient for lung cancer and even steeper than the social class gradient for tuberculosis [9]. The jury remains out on how much, if any, of the ethnic diversity in vital capacity is due to genetic differences.

The excellent review reported by BRAUN et al. [2] makes clear that this debate is not new, but the use of separate racial standards for lung function, as recommended by Prof. Quanjer among others, has strongly influenced clinical practice to view lower vital capacity among these ethnic groups as "normal". If we have made an original contribution to this debate it was in showing that the lower lung function seen among African Americans is associated with exactly the same loss of life expectancy as it would have been associated

Received: Oct 152013 | Accepted after revision: Nov 082013

Conflict of interest: None declared.

Copyright @ERS 2014 
with among white Americans [3]. We conclude that, at least when using lung function to assess prognosis, ethnically adjusted lung function norms should not be used, a conclusion correctly reported by BRAUN et al. [2] .

Our observation was that the norms provided for white Americans gave the correct prediction of mortality in the African American population while the ethnically specific norm did not. Using ethnically specific norms required first an ethnic correction for lung function and then an ethnic correction for mortality in order to undo the effects of the first correction; the alternative was to use no ethnic correction at all. Prof. Quanjer's objection, that this blurs the distinction between causal and non-causal associations, would apply equally to his own assertion of an association between race and lung function when only adjusting for age, sex and height - and is anyway irrelevant because we are not asserting a specific causal association. Our argument is closer to the argument that led astronomers to move from an earth centred universe to a sun centred solar system. The description of the relation between the planets was just a lot simpler if they were assumed to be going around the sun. The development of Newtonian physics to explain these movements did not come for another two centuries.

One issue that is frequently raised in explaining the association of lung function with ethnicity is the varying ratio of trunk length to leg length. The argument runs that if adjustment is made on total height, the proportion of this that is associated with the thoracic height might be expected to have an influence on lung volumes. The potential problem was first raised by HUTCHINSON [10] in his original description of the vital capacity. He thought that it probably made no difference, but others have disagreed since, and it is true that the ratio of trunk length to leg length is systematically different in African compared with white Americans. Analysis of the National Health and Nutrition Examination Survey has explained almost half of the difference in forced vital capacity (FVC) between races in terms of differences in sitting height and much less of this in terms of social factors [11, 12]. In our analysis of the ARIC (Atherosclerosis Risk in Communities) study, however, although we showed the expected difference in relative leg length between the two ethnic groups, we also showed that adjusting for this made very little difference to the contrast in lung function [3]. In either case, differences in relative leg length cannot be quoted as evidence that the difference in lung function between ethnic groups must be due to genetic differences, as there is strong evidence in ethnically homogeneous populations that relative leg length is strongly associated with socioeconomic position in early life $[13,14]$.

Our conclusions have implications both for clinical practice and for research and policy. In clinical practice there is still a role for the kind of norms that Prof. Quanjer has provided [15], used in conjunction with clinical history and other information. For deciding how likely it is that there is a specific pathological process causing loss of vital capacity, specific ethnic norms are useful when used in conjunction with other clinical information, though they are not by themselves diagnostic. When assessing severity or prognosis they should not be used. They should also not be used in epidemiological studies to disguise potential social and environmental disadvantage.

We conclude that the low vital capacity seen in some ethnic groups is not "normal" and is a potential explanation for the very high mortality rates attributed to COPD in low income countries. Some explanation for this is therefore urgently needed, and changes in policy and practice are required to address what is now the third commonest cause of death in the world, and one that specifically picks out the world's poorest populations [16].

\section{Acknowledgement from the editor}

A sentence from a recent editorial by Prof. Quanjer published in the European Respiratory Journal [1] could be interpreted as inappropriate and misleading when read out of context. In an attempt to correct this, Prof. Quanjer has already published an author correction in the journal [17] and we have given space for an editorial in the present issue of the journal in which Prof. Burney and Dr Hooper express their views for our readership.

\section{Postscript from Prof. Quanjer}

It is unfortunate that Prof. Burney and Dr Hooper appear to have misinterpreted my editorial, since my only criticism of their views was in relation to their conclusion that the low FVC explains the higher mortality rate of African Americans. This was based on the simple and unarguable grounds that correlation does not prove causality, an issue yet to be addressed. I think we are all agreed that further work is required to clarify the nature and significance of ethnic differences in spirometry and respiratory morbidity, if we are to improve both clinical practice and public health policy in this field.

\section{References}

Quanjer PH. Lung function, race and ethnicity: a conundrum. Eur Respir J 2013; 41: 1249-1251.

Braun L, Wolfgang M, Dickersin K. Defining race/ethnicity and explaining difference in research studies on lung function. Eur Respir J 2013; 41: 1362-1370. 
Burney PGJ, Hooper RJ. The use of ethnically specific norms for ventilatory function in African-American and white populations. Int J Epidemiol 2012; 41: 782-790.

4 Nassir R, Kosoy R, Tian C, et al. An ancestry informative marker set for determining continental origin: validation and extension using human genome diversity panels. BMC Genetics 2009; 10: 39.

$5 \quad$ Kumar R, Seibold M, Aldrich M, et al. Genetic ancestry in lung-function predictions. N Engl J Med 2010; $363: 321$.

6 Brehm JM, Acosta-Perez E, Klei L, et al. African ancestry and lung function in Puerto Rican children. J Allergy Clin Immunol 2012; 129: 1484-1490.

7 Hughes M, Hertel BR. The significance of color remains: a study of life chances, mate selection, and ethnic consciousness among Black Americans. Social Forces 1990; 68: 1105-1120.

8 Goodman N, Lane RE, Rampling SB. Chronic bronchitis: an introductory examination of existing data. BMJ 1953; 2: $237-243$.

9 Great Britain. Office of Population Censuses and Surveys. Occupational Mortality: The Registrar General's Decennial Supplement for Great Britain, 1979-80, 1982-83. London, H.M. Stationery Office, 1986.

10 Hutchinson J. On the capacity of the lungs, and on the respiratory functions, with a view of establishing a precise and easy method of detecting disease by the spirometer. Medico-Chirurgical Transactions 1846; 29: $137-252$.

11 Harik-Khan RI, Fleg JL, Muller DC, et al. The effect of anthropometric and socioeconomic factors on the racial difference in lung function. Am J Respir Crit Care Med 2001; 164: 1647-1654.

12 Harik-Khan R, Muller D, Wise R. Racial difference in lung function in African-American and White children: effect of anthropometric, socioeconomic, nutritional, and environmental factors. Am J Epidemiol 2004; 160: 893-900.

13 Gunnell DJ, Davey Smith G, Frankel S, et al. Childhood leg length and adult mortality: follow up of the Carnegie (Boyd Orr) Survey of Diet and Health in Pre-war Britain. J Epidemiol Community Health 1998; 52: $142-152$.

14 Wadsworth MEJ, Hardy RJ, Paul AA, et al. Leg and trunk length at 43 years in relation to childhood health, diet and family circumstances; evidence from the 1946 national birth cohort. Int J Epidemiol 2002; 31: 383-390.

15 Quanjer PH, Stanojevic S, Cole TJ, et al. Multi-ethnic reference values for spirometry for the 3-95 year age range: the global lung function 2012 equations. Eur Respir J 2012; 40: 1324-1343.

16 Lozano R, Naghavi M, Foreman K, et al. Global and regional mortality from 235 causes of death for 20 age groups in 1990 and 2010: a systematic analysis for the Global Burden of Disease Study 2010. Lancet 2013; 380: $2095-2128$.

Quanjer PH. Author Correction. Lung function, race and ethnicity: a conundrum. Eur Respir J 2013; 42 : 1162. 